\title{
La planificación del paisaje a escala local en Euskadi. Casos prácticos de planes de acción del paisaje: metodología, participación, conclusiones y reflexiones
}

\author{
Zaro Borda, N ${ }^{* 1}$; Lozano Valencia, P.J. ${ }^{2}$; Etxaniz Millán, I. ; Latasa Zaballo, I. ${ }^{2}$; \\ Muzas Mártinez de Luna, J. ${ }^{2}$
}

${ }^{1}$ Sigi-Saga, Estudios Territoriales/Lurralde Azterlanak. C/Vidangoz 2 Bajo; 31014 Pamplona-Iruñea. nzaro@sigisaga.com; ietxaniz@sigisaga.com

${ }^{2}$ Departamento de Geografia, Prehistoria y Arqueología. Universidad del País Vasco/Euskal Herriko Unibertsitatea. C/ Tomás y Valiente s/n; 01006 Vitoria-Gasteiz.

pedrojose.lozano@ehu.eus; itxaro.latasa@ehu.eus; jmuzas001@ikasle.ehu.eus

*Autora para correspondencia: nzaro@sigisaga.com

\section{Resumen}

Con fecha 21 de julio de 2009, el Gobierno Vasco se adhirió al Convenio Europeo del Paisaje (CEP), aprobado por el Consejo de Europa el 20 de octubre de 2000. Hasta la fecha, las acciones más destacadas han sido la elaboración de varios catálogos de paisaje (a escala de Área Funcional) y, desde 2014, la elaboración de los Planes de Acción del Paisaje (PAP) a una escala local o municipal. La visión y metodología del PAP de la Villa de Amurrio está adaptada a la concepción del CEP, que cambia notablemente la idea de paisaje establecida previamente. En este nuevo contexto, el paisaje pasa de ser una mera construcción científico-técnica o una realidad compleja a partir de elementos y relaciones, a ser también una realidad percibida, sentida e identificada con la población que lo habita, vive, percibe y lo considera no sólo como su ámbito vital, sino como su marco de referencia e identitario. En este PAP se aborda el estudio de la totalidad del territorio del municipio, se definen las unidades de paisaje, los objetivos y las directrices de gestión generales. A partir de ahí, se identifican las áreas de interés paisajístico sobre las que se va a trabajar a las que se asocian unos objetivos de calidad paisajística específicos, con sus correspondientes medidas de actuación. En todo el proceso, la participación ciudadana es fundamental. Con esta comunicación se pretende reflexionar y poner a disposición de la comunidad académica y científica tanto la metodología utilizada como las estrategias de gobernanza más adecuadas.

Palabras clave: directrices de paisaje, medidas de paisaje, ordenación del territorio, participación social, unidades de paisaje. 


\section{Introducción}

El Convenio Europeo del Paisaje (CEP) fue presentado por el Consejo de Europa en Florencia en el año 2000. A día de hoy, son más de 30 los países que lo han ratificado, entre ellos España, donde entró en vigor el 1 de marzo de 2008. Son las Comunidades Autónomas (CCAA) quienes deben desarrollar los principios y reglamentaciones del CEP, siendo Cataluña la primera y más destacada hasta la fecha; no en vano ha desarrollado una ley ad hoc y un profuso trabajo de generación de catálogos de paisaje (CCPP) (Nogué y Sala, 2008). También Valencia y Galicia disponen de una legislación que ha creado instrumentos específicos para la protección, ordenación y gestión del paisaje. Por otra parte, hay comunidades que han creado una ley, pero sin desarrollo posterior; y otras que, no habiendo generado una ley específica, han desarrollado diversos instrumentos, caso de Madrid, La Rioja, Andalucía, Murcia, Aragón y Canarias. (Sabaté y Vera, 2008).

El País Vasco viene desarrollando desde 2005 los Catálogos de Paisajes Singulares y Sobresalientes que, aunque han venido siendo utilizados como condicionante superpuesto dentro de los procesos y políticas territoriales, no son acordes a uno de los objetivos esenciales del CEP, que aboga por una política de paisaje que incluya todo el territorio y todo tipo de paisajes, no sólo los más notables. Ello trató de ser corregido por el Gobierno Vasco (GV) con la resolución de 28 de marzo de 2011, donde se presentó el anteproyecto de Ley del Paisaje del País Vasco (ALPPV). Posteriormente, dicho documento no llegó a ser aprobado en forma de ley, sino de decreto, en concreto: Decreto 90/2014, de 3 de junio, sobre protección, gestión y ordenación del paisaje del País Vasco. En paralelo, el GV puso en marcha el proceso de redacción de los tres primeros catálogos de paisaje, para cuya elaboración se eligieron, como unidad territorial de base, las áreas funcionales, a una escala que fluctúa entre el 1:50.000 y 1:25.000, cuestión clave para el desarrollo de los dictámenes de los catálogos dentro de las propias políticas territoriales (Gobierno Vasco, 2011).

Por otra parte y, con la misma filosofía de integración del paisaje dentro de las distintas figuras relacionadas con la OT o, como es el caso, con los planes urbanísticos, el GV también puso en marcha desde el verano de 2014 una línea de subvención a la que podrían acogerse los distintos municipios de la CAPV para subvencionar planes concretos de conservación, ordenación, gestión y recuperación del paisaje dentro de esta escala local o municipal. La principal debilidad ha sido la inexistencia previa de un decálogo o documento normativo o director que iluminará la filosofía pero, sobre todo, la metodología a la hora de abordar estos PAP. En el presente trabajo se aborda el desarrollo de uno de estos planes dentro del Municipio de Amurrio.

Desde tal perspectiva, pretendemos cumplir los siguientes objetivos:

a. Exponer a la comunidad técnica y científica un ejemplo concreto de desarrollo de uno de estos planes; el PAP de la Villa de Amurrio.

b. Exponer, de igual manera, la metodología general empleada para el desarrollo de esta figura de planificación de cara a su crítica y cotejo por la mencionada comunidad científico/técnica.

c. Dicha exposición pretende abrir un debate para la discusión sobre las mejo- 
res metodologías y herramientas a la hora de realizar estos PAP, tanto dentro de los procesos de conservación, ordenación y gestión de los paisajes a escala local, como de los necesarios procesos de participación ciudadana.

d. Centrar nuestras propuestas dentro de una de las unidades determinadas para contar con un ejemplo más concreto y acorde con la filosofía del presente congreso.

e. Proporcionar un marco metodológico general que pueda servir, a futuro, para que otros equipos puedan utilizarlo e implementarlo en otras unidades territoriales.

f. Generar un documento que ayude a la toma de decisiones a las autoridades políticas y técnicas con competencias en esta materia.

\section{Situación}

El municipio de Amurrio se sitúa al noroeste de la provincia de Álava dentro de la comarca de Aiara-Ayala. Comparte dicha comarca geográfico-histórica con los municipios de: Aiara-Ayala, Artziniega, Laudio-Llodio, Okondo y Orduña. En la actualidad cuenta con alrededor de 10.068 habitantes y, aunque pueda parecer que su población es modesta, fuera del ámbito de Vitoria-Gasteiz, la capital de la provincia,
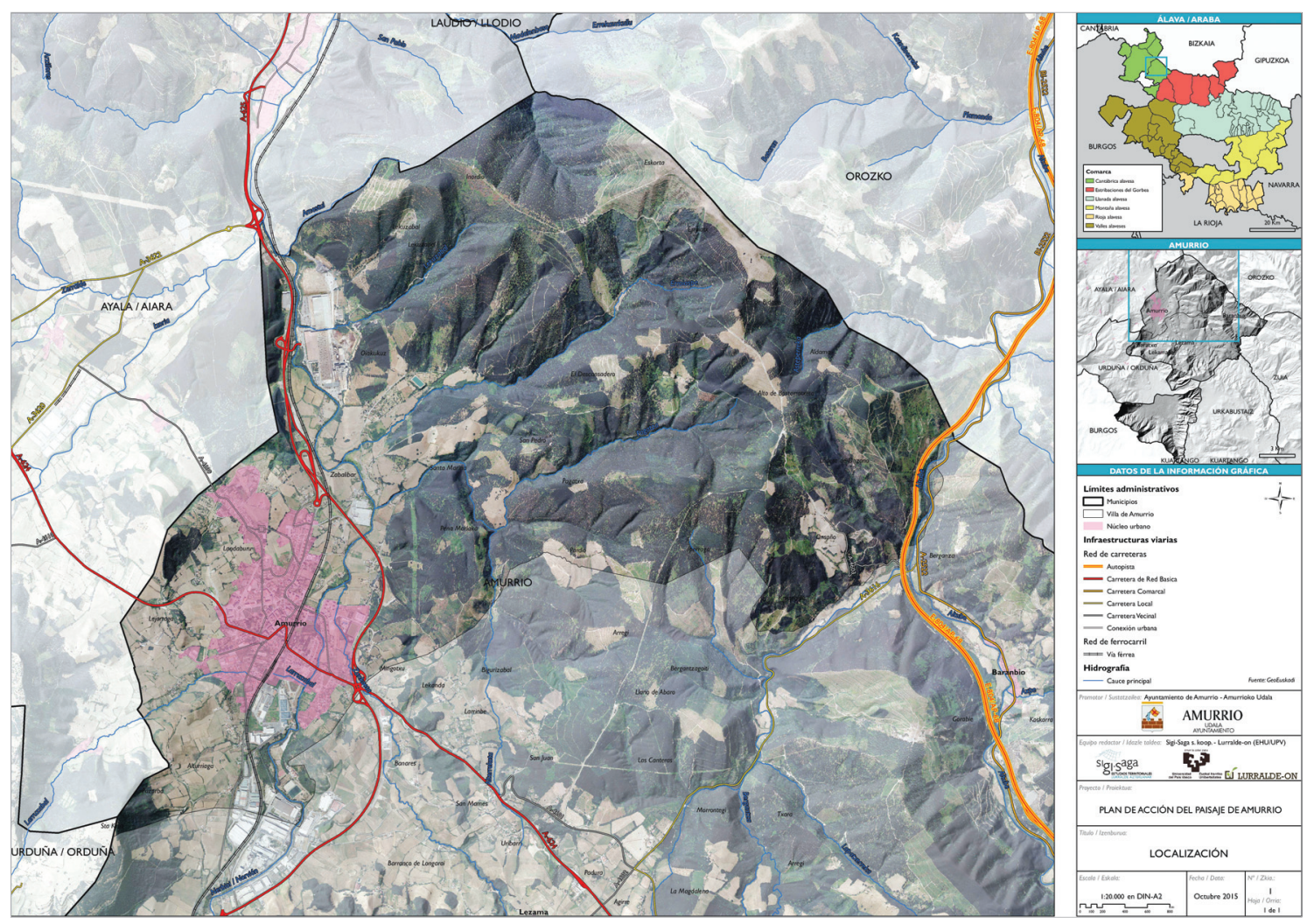

Figura 1. Localización del Área de trabajo. Fuente: Elaboración propia. 
se configura como uno de los municipios que soporta más población. En concreto, es la tercera entidad de población por detrás de la capital y de otro municipio de la misma comarca; Laudio-Llodio. Por su parte, cuenta con una extensión bastante amplia (si se estima el tamaño de los distintos municipios de la provincia de Álava); de $96,4 \mathrm{~km} 2$ a la vez que muestra un territorio y unos paisajes ciertamente notables y, en muchos sectores, con gran calidad.

Uno de los aspectos más interesantes del municipio es que muestra una estructura territorial ciertamente original puesto que se encuentra dividido en hasta 10 concejos diferentes. Éstos son de sur a norte: Delika, Tertanga, Artomaña, Aloria, Lezama, Lekamana, Saratxo, Larrimbe, Barambio y el propio Amurrio. Este último es el que aglutina la mayor cantidad de población y la mayor extensión, en concreto unos $25 \mathrm{~km}^{2}$. Es precisamente este concejo donde se centró el desarrollo del plan de acción del paisaje homónimo.

Como en la mayoría de los municipios industriales de la CAPV, desde mediados del pasado siglo se dio lugar a una radical transformación del paisaje a partir de unos importantes procesos de urbanización e industrialización. Junto a ello, una de las principales industrias del territorio pasó a ser la papelera. A partir de su pujanza y de un proceso paralelo de abandono rural, gran parte de lo que antes eran campos de cultivo o pastos para la ganadería, fueron siendo ocupados sistemáticamente por bosques de repoblación con especies exóticas, fundamentalmente pino de Monterrey (Pinus radiata). Así, las zonas topográficamente más amables: fondos de valles fluviales, fueron fosilizadas y cementadas por los suelos urbanos e industriales mientras las laderas medias y altas lo fueron por las mencionadas repoblaciones. Con ello, a día de hoy, se puede constatar que el suelo urbanizado (viviendas, actividad económica industrial y de servicios y sistemas generales) es del $5,32 \mathrm{~km}^{2}$, suponiendo un $5,80 \%$ del suelo total. Teniendo en cuenta que de espacios libres de uso público dentro del suelo urbano existen $32,39 \mathrm{Ha}$ y de cauces fluviales $29,52 \mathrm{Ha}$, aunque se encuentren dentro del espacio urbanizado, el 11,28\% de dicho espacio se referiría a espacios, en gran medida, no cementados u ocupados por lo que podemos definirlos como infraestructura verde urbana.

Por otra parte y a la vista de las distintas categorías de suelos no urbanizables (Udalplan, 2014), se puede observar que existe una gran proporción del mismo que aparece englobado bajo distintas categorías que cuentan con una vocación proteccionista; especial protección 1564,26 Ha, mientras que para la protección de aguas superficiales existen 230,39 Ha. En lo que respecta a la vocación forestal, éste es el capítulo más importante del suelo no urbanizable, por debajo de "sin vocación": $1.614,53$ y $5.256,18 \mathrm{Ha}$ respectivamente.

Sin embargo, en lo que respecta al concejo de Amurrio, la superficie urbana supone un $11,82 \%$ del territorio, mientras que el paisaje agrario ( $\sin$ forestal) supone un $25,03 \%$ y el resto, fundamentalmente paisaje forestal y natural supone un $63,15 \%$.

Esta unidad presenta una clara dualidad entre una importante actividad industrial muy consolidada y de gran tradición junto a la ubicación del núcleo urbano más importante entorno al río, en concreto en el fondo del valle de Amurrio. En realidad, las circunstancias topográficas, con un valle fluvial de fondo plano y amable, han con- 
dicionado secularmente el asentamiento no sólo urbano o productivo, sino también de las infraestructuras de comunicación y las dotaciones más importantes. No obstante, aunque la presión antrópica ha sido fuerte, dentro de la misma unidad del fondo de valle todavía se encuentran importantes retazos de campiña atlántica. A su vez, ha dado lugar a un hábitat disperso, típico de la zona atlántica del País Vasco, a modo de caseríos diseminados que, un día, supusieron verdaderas unidades de producción y que, hasta cierto punto, algunos siguen siéndolo. En los últimos años, no obstante, en la mayor parte de los mismos ha sido abandonada esa unidad de producción, manteniendo una gran parte de los mismos, solamente la función de vivienda.

Aparte del mantenimiento de estos valores ambientales y productivos, el paisaje agrario típico de la campiña atlántica mantiene un rico patrimonio arquitectónico (caseríos), étnico-histórico (caleros, hornos, edificios auxiliares, muros de piedra seca), naturales (setos vivos, bosquetes...) e incluso inmateriales en forma de costumbres, celebraciones, fiestas, romerías, tradiciones...

Conforme nos alejamos del río Nervión observamos una transición rural-forestal. En las laderas medias que rodean el fondo del valle se instalan diferentes tipos de bosques que vuelven a presentar una dicotomía entre retazos y zonas de repoblación y otros que mantienen rodales de vegetación autóctona. Las superficies que bajo titularidad privada siguen apostando por bosques de exóticas con ciclos cortos de corta y una explotación intensiva ocupan hasta en un $82 \%$.

En las partes más elevadas (cordales montañosos de media altitud) existen importantes caminos y miradores que acercan al ciudadano hacia la contemplación, el paseo y el disfrute del paisaje interno y externo.

En cualquier caso, Amurrio, muestra un estado del paisaje, en general, bastante óptimo y aceptable. Sus bajas densidades poblacionales (con excepción del núcleo principal) pero sobre todo su configuración territorial y paisajística ha hecho que su calidad sea ciertamente notable y que, en gran medida (32\%), esté catalogado dentro del Catálogo Vasco de Paisajes Notables y Sobresalientes.

\section{Metodología}

La metodología utilizada se centra en el análisis, caracterización y evaluación del paisaje y dedica especial atención a la participación social como elemento y herramienta fundamental, vinculada a la percepción de la población conforme a la propuesta del CEP. La aplicación de dicha metodología al estudio de los paisajes del concejo de Amurrio está produciendo sus primeros resultados en el contexto de la ordenación del paisaje a escala local, cuyo objetivo primordial es contribuir a la resolución de problemas o necesidades planteadas por la sociedad. Si bien se toma como referencia la metodología de los Catálogos de Paisaje de la Comunidad Autónoma de Catalunya (Nogué y Sala, 2006) y la de los de la Comunidad Autónoma del País Vasco (Gobierno Vasco, 2011), se apuesta por un método de valoración e implementación novedoso donde, de forma no sólo cualitativa, se aborde la valoración de cada unidad de paisaje. 
El método se estructura en tres etapas (con sus correspondientes subetapas): análisis territorial, planificación del paisaje y gestión del propio paisaje. En lo que respecta al análisis territorial, una vez delimitado el ámbito de trabajo (el Concejo de Amurrio), se pasó a la recopilación, generación, sistematización y clasificación de la información, cara a la realización del análisis preliminar. Teniendo en consideración las debilidades, amenazas, fortalezas y oportunidades del territorio, se conformó un primer diagnóstico y se elaboró una cartografía básica a escala local. En paralelo, se configuró la lista de agentes y el grupo de trabajo y discusión que iban a participar en el proceso. A todos los agentes relevantes se les pasó un cuestionario de entrevista con más de 40 preguntas. A partir de ese cuestionario y de las respuestas obtenidas se abordó la realización de una encuesta de valoración con un tamaño muestral suficientemente robusto para aglutinar y representar al conjunto de la población y sus anhelos respecto al territorio.

Con todo ello, el equipo redactor abordó la tarea de definición de las unidades de paisaje a partir de elementos primarios como la estructura fisiográfica (litología, tectónica, evolución geológico-geomorfológica, procesos y formas), textura (usos del suelo, vegetación, parcelario, elementos antrópicos) historia y tradición (evolución de usos, diacronía, historia de las comunidades que transformaron el paisaje, usos y costumbres, tradiciones), visibilidad (potencial de vistas, exposición visual, fragilidad, cuencas visuales, definición de miradores), y percepción y valoración del paisa-
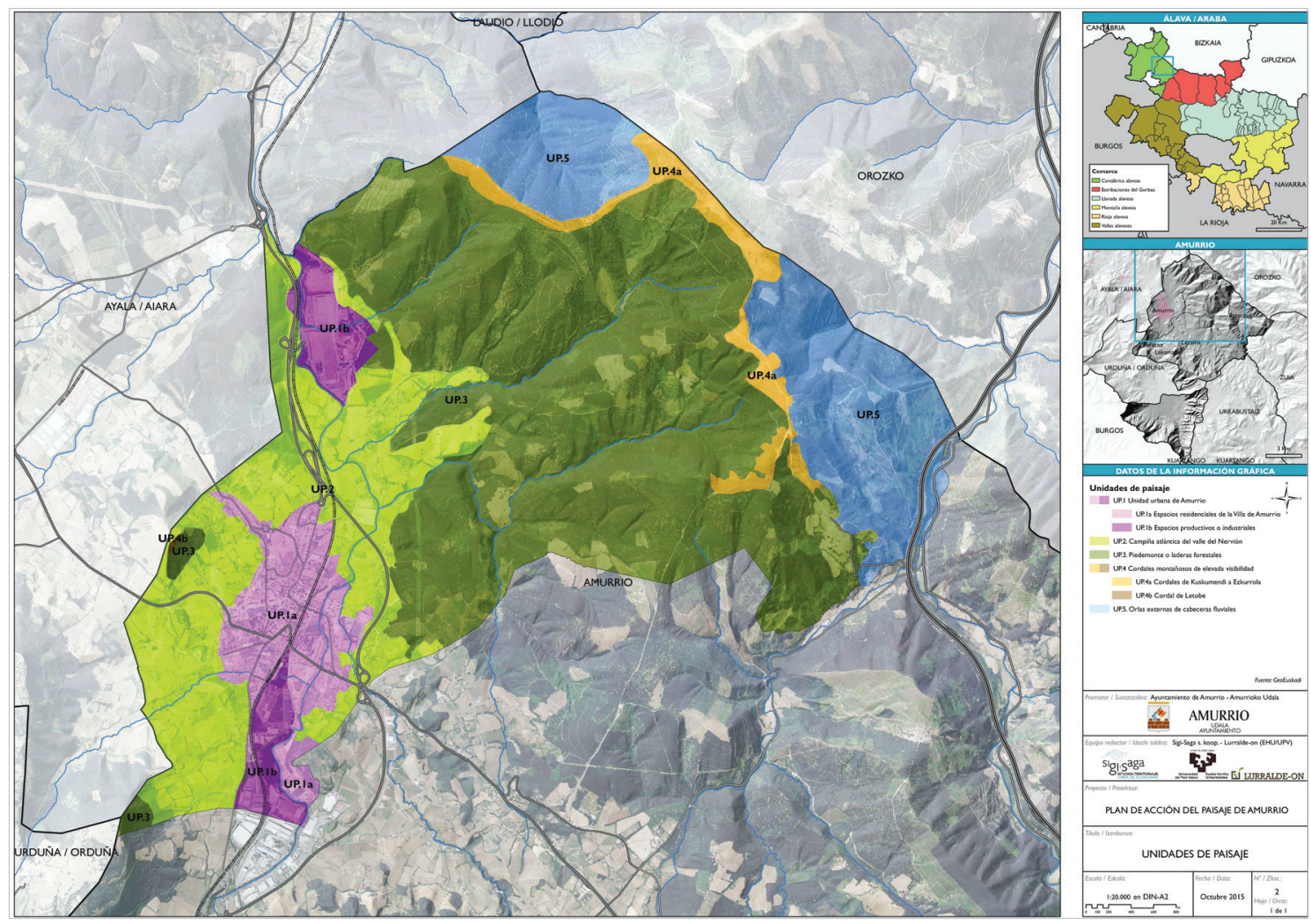

Figura 2. Unidades de Paisaje. Fuente: Elaboración propia. 
je (recogida y síntesis de la información derivada de la encuesta general, de las entrevistas a los agentes y de los resultados de los los grupos de trabajo y discusión ). Las unidades primarias así definidas fueron recogidas en cinco mapas temáticos (unidades morfoestructurales, unidades texturales sintéticas, unidades diacrónicas de evolución y gestión, grandes unidades visuales, unidades sociales y perceptivas), cuya síntesis dio lugar a un mapa inicial de unidades primarias del paisaje.

Posteriormente, al primer mapa de unidades primarias se le añadieron los resultados del análisis de los elementos secundarios del paisaje: riesgos naturales (riadas, movimientos en masa, riesgos antrópicos), conectividad paisajística y ecológica (red de corredores ecológicos del País Vasco, lugares potenciales para la visualización de flora y fauna), fenología y color (evolución fenológica de vegetación y cultivos, colores, formas), elementos climáticos (meteoros, evolución de la sucesión natural de los distintos tipos de tiempo a lo largo del año), elementos singulares (paisajes notables y sobresalientes, espacios o paisajes protegidos, patrimonio histórico-arquitectónico-etnográfico-cultural), y evolución y tendencias de futuro (agentes, presiones, efectos, impactos y prognosis). Todo ello, añadido al análisis y al trabajo de SIG, dio lugar a las unidades secundarias o de matización, que fueron sometidas a la crítica del grupo de trabajo y discusión, así como de expertos en la materia dentro y fuera de la comunidad científica y del territorio de análisis. Con todo ello se ejecutó el mapa de unidades de paisaje del Concejo de Amurrio.

Una vez definidas dichas unidades, se repitió el protocolo operativo específico para cada una de ellas, abriéndose la segunda etapa: la de planificación del paisaje. Se retomó la información ya compilada y analizada, y se detectaron los posibles vacíos para generarla ad hoc y, posteriormente, abordar las debilidades, amenazas, fortalezas y oportunidades cara a emitir su diagnóstico paisajístico. Para ello se generaron las tablas DAFO de síntesis y la definición de objetivos para corregir las debilidades, soslayar las amenazas, mantener las fortalezas, aprovechar las oportunidades y asegurar las mejoras. A partir del análisis del marco jurídico-administrativo y una vez medido el grado de compatibilidad entre los objetivos propuestos, se pasó a la determinación de las alternativas o directrices del paisaje divididas en las mismas 5 categorías de los objetivos, de manera que exista una relación directa e inequívoca para cada uno de los puntos del diagnóstico con respecto a su objetivo u objetivos y su directriz o directrices. Con ello, el gestor puede comprobar la relación de cada uno de los aspectos diagnosticados y su formulación como objetivo, alternativa o directriz. Junto a estos procesos y subetapas, se realizó una división del territorio, en general, y de cada una de las unidades, en particular, en tres categorías: paisajes de protección, paisajes de planificación y paisajes de gestión, elaborando para cada una de ellas las medidas generales y los pasos a dar para su correcta gestión.

Por último, tras cotejar los resultados obtenidos con el grupo de trabajo y discusión y un doble consejo de expertos (de dentro y fuera del Concejo de Amurrio), se pasó a generar las fichas para cada una de las unidades, dando por concluido el proceso de planificación del paisaje. El protocolo se cerró con la entrega de la documentación al ayuntamiento y al GV para su eventual puesta en marcha e implementación. 


\section{Resultados y Discusión}

Debido a que el espacio para la comunicación es escaso, hemos decido centrarnos en una de las unidades. En este caso, la que mejor se adecúa a la propia idiosincrasia del evento es la unidad 3. Ésta fue denominada, a partir de los procesos de participación ciudadana como: "piedemonte o ladera forestal" y es sobre la que se va a basar este apartado de resultados y discusión.

Bajo la denominación de piedemonte o laderas forestales se han incluido los espacios ubicados entre la campiña atlántica y los cordales montañosos de elevada visibilidad. En esta unidad predomina la componente forestal y en ella se ubican también algunos núcleos rurales dispersos con sus correspondientes entornos agro-ganaderos. Esta unidad engloba la zona central del término y es la que comprende una mayor superficie. Los criterios para su delimitación han sido tres:

- Espacios forestales que unen las áreas de alta visibilidad (cordal montañoso) con el fondo de valle (Campiña Atlántica y Espacios o Paisajes Urbanizados).

- Espacios donde domina la cobertura forestal y, dentro de ésta, las plantaciones silvícolas con especies alóctonas.

— Topografía de vertiente con pendientes que llegan a ser elevadas.

Como en cualquier trabajo de ordenación territorial o paisajística, lo primero y, a resultas del diagnóstico técnico y ciudadano, es aquilitar un conjunto de objetivos generales que intenten paliar las debilidades detectadas o que intenten aprovechar las fortalezas que la unidad pueda presentar. A continuación se desarrollan los objetivos específicos u operacionales que cuentan con un mayor grado de concreción.

Los objetivos generales planteados para todo el paisaje del concejo (OG) son los siguientes:

- OG.1 Conservar el paisaje.

- OG.2 Mejorar el paisaje del ámbito urbano.

- OG.3 Mantener, mejorar y restaurar el paisaje del ámbito rural.

- OG.4 Articular armónicamente el paisaje.

- OG.5 Recuperar el paisaje.

- OG.6 Poner en valor y proteger el paisaje.

— OG.7 Atribuir valor o crear paisaje.

Los objetivos específicos y operacionales (OE) de calidad paisajística asignados a esta unidad han sido los siguientes:

- OE.1.3. Favorecer las actividades que contribuyan a la conservación de los valores ambientales de los entornos forestales.

- OE.3.1. Mantener y restaurar los paisajes y elementos paisajísticos que confieren heterogeneidad a la unidad.

- OE.3.2. Potenciar la mejora y restauración ambiental y paisajística en ámbito rural.

- OE.4.2. Acometer actuaciones que favorezcan la transición paisajística ar- 
mónica entre diferentes tipos de unidades paisajísticas o ambientales.

- OE.6.2. Promover actuaciones que potencien el carácter multifuncional del espacio.

Los objetivos específicos se identifican mediante el acrónimo "OE", seguido de un primer dígito que se corresponde con el del objetivo general en el que se incluye y un segundo número correlativo que lo diferencia del resto. Una vez planteados los objetivos que muestran, simplemente, la voluntad de cambio, mejora o mantenimiento de las condiciones óptimas, es necesario plantear las medidas que conduzcan al cumplimiento de cada uno de los objetivos planteados. Para ello, como mínimo, se plantea una alternativa para cada objetivo formulado.

Las medidas (ME) han sido orientadas a la consecución de los objetivos de calidad paisajística definidos para cada área de especial interés paisajístico. Se citan algunas de las relacionadas con la unidad de paisaje 3. Los tres primeros dígitos se corresponden con los OE y el cuarto con las medidas asociadas a cada uno de ellos:

- ME.4.2.1.1 Naturalización de los bordes de la servidumbre del oleoducto.

- ME.4.2.1.2 Plantación de pequeños bosquetes arbustivos y de frondosas en la primera línea de borde de servidumbre.

- ME.6.2.2.3 Potenciación de iniciativas orientadas a promover el uso ganadero del corredor, que contribuyan, a su vez, a facilitar el estado de mantenimiento del mismo.

- ME.3.1.2.1 Plantación con varias especies de frondosas autóctonas en el terreno municipal.

- ME.3.1.2.3 Creación de un arboretum a partir de una plantación diseminada de especies arbóreas y arbustivas autóctonas.

- ME.1.3.1.1 Acometer actuaciones forestales encaminadas a facilitar la creación y mantenimiento de corredores ecológicos entre bosquetes y rodales de vegetación autóctona.

— ME.3.2.2.1 Puesta en marcha de una campaña de información, sensibilización e incentivación dirigida a los propietarios forestales particulares para que integren criterios paisajísticos y consideren la sustitución de especies forestales.

- ME.3.2.2.2 Implementar medidas de sustitución progresiva de especies de coníferas por frondosas (preferiblemente autóctonas) en los terrenos de titularidad municipal.

— ME.4.2.2.1 Aplicación de criterios de integración paisajística entre las diferentes unidades ambientales y ecotonos existentes.

- ME.6.2.4.1 Redacción de una ordenanza municipal para la regulación de la gestión-utilización de los recursos micológicos.

— ME.6.2.4.2 Mantenimiento y potenciación de rasos con fines ganaderos y de disfrute del paisaje.

De cara a facilitar la gestión, se deben tener en cuenta una serie de cuestiones que, 
a continuación se detallan y se han tenido en cuenta a la hora de desarrollar las medidas:

- Cada una de las acciones debe estar bien explicada y determinada.

- Cada una de las acciones debe estar bien situada cronológicamente, de manera que se especifique su periodo de vigencia o desarrollo.

- Cada una de las acciones debe determinar un modo o modelo de medición del éxito de su consecución, de manera que se pueden establecer una batería de indicadores fácilmente evaluables.

Se han seleccionado además una serie de indicadores para llevar a cabo el seguimiento del PAP. Los indicadores deben ser instrumentos funcionales que permitan evaluar la efectividad de las acciones establecidas para alcanzar los objetivos de calidad paisajística fijados. Cada medida o acción tiene definidos los resultados esperados con su ejecución y su propio indicador o sistema de medición asociado que permita evaluarla. Los indicadores, así como los resultados, se recogen en fichas de medidas elaboradas.

El objetivo del seguimiento debería ser:

- Realizar un análisis y seguimiento del grado de implantación del plan.

- Evaluar de los resultados obtenidos.

- Dar difusión social a las acciones ejecutadas.

- Tratar de atraer nuevos agentes que se impliquen en el desarrollo del plan.

- Buscar fuentes de financiación que permitan abordar las medidas propuestas.

- Redirigir las actuaciones ejecutadas o en ejecución y definir las previstas en el corto, medio y largo plazo.

\section{Conclusiones}

Las principales conclusiones y reflexiones que se extraen de la elaboración de este PAP y de las reuniones de presentación de otros, son las siguientes:

1. La interpretación conceptual y metodológica abierta para la elaboración de estos planes ha dado lugar a visiones muy dispares a la hora de abordar los mismos.

2. La ausencia de un catálogo de paisaje en el ámbito de estudio hace necesario realizar un análisis paisajístico previo que además puede dar lugar a la heterogeneidad en la concepción, análisis y desarrollo de estos planes, derivada de la ausencia de un instrumento de escala mayor.

3. El desarrollo del plan está supeditado, en cierta medida, a la propuesta presentada por los municipios a la convocatoria de subvenciones, ya que la definición de las medidas se centra en esta Áreas de Especial Interés Paisajístico y no en la totalidad de las Unidades de Paisaje.

4. El comprometimiento y la participación activa de la población en temas tan 
etéreos es complicada, por lo que es conveniente buscar vías alternativas de participación.

5. La unidad 3 que ha sido el objeto de esta comunicación muestra potencialidades muy remarcables como sus valores naturales y patrimoniales, por el contrario, muestra una cierta homogeneidad centrada en las repoblaciones forestales con especies exóticas que homogenizan, en exceso, el paisaje. Debido a estas cuestiones y a la incertidumbre registrada en torno a la aparición de enfermedades que diezman las mismas y lo voluble y bajo del precio de la madera, es necesario poner en marcha medidas de diversificación, mayor heterogeneidad y naturalidad dentro de esta unidad.

\section{Bibliografía}

Gobierno Vasco; 2011. Elaboración de los catálogos y directrices de paisaje. Presentación. http://www.ingurumena.ejgv.euskadi.net/r49-paisaia/es/

Gobierno Vasco; 2013. Políticas territoriales en la Comunidad Autónoma del País Vasco: http://www.ingurumena.ejgv.euskadi.net/r49-578/es/

Nogué, J. y Sala, P.; 2006. Prototipo de Catálogo de Paisaje. Bases conceptuales, metodológicas y procedimentales para la elaboración de los Catálogos del Paisaje de Cataluña. Olot y Barcelona. http://www.catpaisatge.net/fitxers/resumenprototipo.pdf

Nogué, J. y Sala, P.; 2008. El Paisaje en la Ordenación del Territorio. Los catálogos del paisaje de Cataluña. Cuadernos Geográficos, 4,: 69-98.

Sabaté, J. y Vera, J.R.; 2008. Aspectos varios de la implementación de la Convención Europea del Paisaje en el Plan Territorial Especial de Ordenación del Paisaje de Tenerife. Cuadernos Geográficos, 42, 51-67.

Udalplan, 2014; http://www.ingurumena.ejgv.euskadi.eus/r49-udalplan/eu/aa33aWAR/inter facesJSP/index.jsp 
